\title{
Clinical Characteristics and CT Manifestations of 143 Patients With 2019 Novel Coronavirus Disease (COVID-19) in Taizhou City, Zhejiang, China
}

\section{Yani Kuang}

Taizhou Hospital affiliated to Wenzhou Medical University, Zhejiang, China

\section{Susu He}

Taizhou Hospital affiliated to Wenzhou Medical University, Zhejiang, China

Shuangxiang Lin

Taizhou Hospital affiliated to Wenzhou Medical University, Zhejiang, China

\section{Rui Zhu}

Taizhou Hospital affiliated to Wenzhou Medical University, Zhejiang, China

\section{Rongzhen Zhou}

Taizhou Hospital affiliated to Wenzhou Medical University, Zhejiang, China

\section{Shuying Ying}

Taizhou Hospital affiliated to Wenzhou Medical University, Zhejiang, China

\section{Renzhan Li}

Sanmen People's Hospital, Zhejiang, China

\section{Haiyong Lin}

Wenling First People's Hospital, Zhejiang, China

\section{Zhibang Zhang}

Tiantai People's Hospital, Zhejiang, China

\section{Peipei Pang}

Hangzhou GE Healthcare, China

Wenbin Ji ( $\square$ wb.j@163.com )

Taizhou Hospital affiliated to Wenzhou Medical University, Zhejiang, China https://orcid.org/00000002-7667-9430

\section{Research}

Keywords: COVID-19, COVID-19 pneumonia, Clinical characteristics, CT manifestations

Posted Date: June 5th, 2020

DOI: https://doi.org/10.21203/rs.3.rs-32895/v1 
License: (c) (i) This work is licensed under a Creative Commons Attribution 4.0 International License. Read Full License 


\section{Abstract}

Background: In December 2019, the first case of pneumonia associated with the SARS-CoV-2 was found in Wuhan and rapidly spread throughout China, so data are needed on the affected patients. The purpose of our study was to find the clinical manifestations and CT features of COVID-19.

Methods: All patients with COVID-19 in Taizhou city were retrospectively included and divided into nonsevere group and severe group according to the severity of the disease. The clinical manifestations, laboratory examinations and imaging features of COVID-19 patients were analyzed, and the differences between the two groups were compared.

Results: A total of 143 laboratory-confirmed cases were included in the study, including 110 non-severe patients and 33 severe patients. The median age of patients was 47 (range $4-86$ years). Fever $(73.4 \%)$ and cough (63.6\%) were the most common initial clinical symptoms. Between two groups of cases, the results of aspartate transaminase, creatine kinase and lactate dehydrogenase, serum albumin, CR, glomerular filtration rate, amyloid protein A, fibrinogen, calcitonin level and oxygen partial pressure, IL 10 , absolute value of CD3, CD4, CD8 were different, and the difference was statistically significant $(\mathrm{P}<$ 0.05). Therefore, these quantitative indicators can be used to help assess the severity. On admission, the CT showed that the lesions were mostly distributed in the periphery of the lung or subpleural (135 cases $(98 \%))$, and most of lesions presented as patchy ( $81 \%)$, mixed density $(63 \%)$ shadow. Consolidation (68\% vs $41 \%)$, bronchial inflation signs ( $59 \%$ vs $41 \%)$, and bronchiectasis $(71 \%$ vs $39 \%$ ) were more common in the severe group.

Conclusions: Most of the cases of COVID-19 in Taizhou have mild symptoms and no death. In addition to clinical symptoms, some laboratory tests (such as absolute values of CD4 and CD8) and CT findings can be used to assess the severity of the disease.

\section{Background}

In December 2019, the first pneumonia cases of unknown origin were reported in Wuhan[1-3], capital of China's Hubei province. Later, the pathogen was identified as a new enveloped RNA-Beta-coronavirus, which was considered to have developmental similarity with SARS-CoV[4]. WHO named the virus severe acute respiratory syndrome coronavirus 2(SARS-CoV-2), while the disease it caused refers to coronavirus disease(COVID-19)[5, 6].Most of those infected patients presented as acute viral pneumonia, and there is clear evidence of human-to-human transmission[7-10].

On January 30, 2020, WHO declared COVID-19 the sixth public health emergency of international concern (PHEIC).As of March 6, 2020, there were 97,769 laboratory-confirmed cases worldwide[11]. The statistics of SARS-CoV(severe acute respiratory syndrome coronavirus), which appeared in 2003, showed that there were 8,422 infected people[12, 13]. And in 2012, MERS-CoV(middle east respiratory syndrome coronavirus) was also prevalent in the Middle East mainly[14-17]. It's clear that COVID-19 has become a globally widespread disease[11, 18, 19]. 
Due to the people movement during the Spring Festival, many Taizhou residents in Wuhan returned, created opportunities for the virus to spread in Taizhou. Taizhou, a city in Zhejiang province, is listed among the top three cities in the province for the number of confirmed cases. There were a number of studies on the clinical characteristics of patients in Wuhan[20, 21]. Given the rapid spread of COVID-19, we believe that the latest analysis of cases outside Wuhan may help to grasp the overall characteristics of transmission and further determine the clinical characteristics and severity of the disease. Therefore, this paper presents the results of our study on the clinical characteristics and imaging manifestations of COVID-19 infection in Taizhou city, hoping to better understand the characteristics of this disease.

\section{Methods}

\section{Study participants and design}

This retrospective multicenter cohort study was approved by the Institutional Review Board of each participating hospital. Given public health emergency situation, written informed consent was waived. From January 17 to March 11, 2020, patients were consecutively enrolled in four hospitals in Taizhou city. The four hospitals include a municipal hospital and three county-level hospitals, namely Taizhou Hospital Enze district, Wenling First People's Hospital, Sanmen People's Hospital and Tiantai People's Hospital. All patients were admitted after laboratory confirmation of COVID-19 infection. The clinical outcomes were monitored up till March 11, 2020, the final date of follow-up.

\section{Data source}

We obtained electronic medical records and data of laboratory-confirmed COVID-19 infections in four hospitals from January 17 to March 11, 2020. The diagnostic criteria were positive tests by high throughput real-time reverse transcription polymerase chain reaction (RT-PCR) of nasal swabs and pharyngeal swabs recommended by the WHO interim guidelines. As all the confirmed diseases in Taizhou were treated in the public health center of Enze district of Taizhou hospital, the medical records of some patients before admission were provided by the doctors in their respective hospitals. The cases from Wenling People's Hospital, Sanmen People's Hospital and Tiantai People's Hospital were sent to the Taizhou Hospital researchers by the participants in the hospital. Only laboratory-confirmed cases were included in the study.

All clinical data were reviewed and extracted by a team of experienced respiratory clinicians in Taizhou hospital. When record the data in an Excel spreadsheet, if the data is missing, a request is made to the hospital where the case is located, and the hospital participant then contacts the attending clinician. We extracted recent exposure history, clinical symptoms or signs, and laboratory examination results on admission from the electronic medical record. Imaging examinations include chest X-ray or computed tomography (CT) of thorax. All patients admitted to the CT were evaluated and reviewed by senior radiologists in Taizhou hospital. Any major differences between two reviewers shall be resolved by 
discussion with the third panel of reviewers. All laboratory examinations were performed according to the patient's clinical care needs, including a complete blood count, blood chemical analysis, coagulation test, assessment of liver and kidney function, as well as electrolytes, c-reactive protein, calcitonin, lactate dehydrogenase, lymphocyte factor assay, blood gas analysis, and creatine kinase measurements.

\section{Study definitions and criteria}

We defined the severity (severe vs. non-severe) of COVID - 19 patients according Diagnosis and Treatment Protocol for Novel Coronavirus Pneumonia( Trial Version 5) released by the National Health Commission\& State Administration of Traditional Chinese Medicine[22]. The severe group included the serious and critical type, while the non-severe group included the mild and moderate types. Mild type was defined as clinical symptoms were mild, and no signs of pneumonia were found on imaging. Moderate type was defined as having symptoms such as fever, respiratory tract symptoms, and the appearance of pneumonia can be seen on imaging. Serious type was defined as satisfying any of the following items:1. Respiratory distress, RR > 30 / min;2.At rest, oxygen saturation $<93 \%$; 3 . Arterial blood oxygen partial pressure $(\mathrm{Pa02})$ /oxygen concentration $(\mathrm{FiO2})<300 \mathrm{mmHg}(1 \mathrm{mmHg}=0.133 \mathrm{KPa})$. Critical type was defined as satisfying any of the following items:1. Respiratory failure and the need for mechanical ventilation;2.Shock;3.Combined with other organ failure requires ICU care.

Date of exposure refers to the earliest date of exposure to a source of transmission (persons in Wuhan or confirmed patients).The incubation period was defined as the time interval between the potential earliest date of exposure to the source of transmission and the earliest date of occurrence of symptoms (i.e., clinical symptoms such as cough and fever).Record the specific contact date (if the date was not clear, it will not be included in the analysis) and calculate the incubation period based on the specific information of the exposure date. Treatment delay indicates the time between symptoms onset and hospitalization. The course of illness indicated the time from symptoms onset to discharge. The discharge time of patients who had been discharged was recorded, and the cases with definite symptom onset date (those with unclear date were not included in the analysis) were included in the course of illness analysis. Body temperature under the armpit greater than or equal to $37.5^{\circ} \mathrm{C}$ defined as fever. Normal values of other laboratory examination indicators are shown in the Table2. 
Table 1

Cinical Characteristics of the Study Patients, According to Disease Severity

Overall Disease severity

$(n=143)$

$\begin{array}{ll}\text { Non-severe } & \text { Severe } \\ (n=110) & (n=33)\end{array}$

PValue

Age(years)

Median(range)

$47.0(4-86)$

$44.5(4-80)$

$55.0(27-$

$<0.05$

$<38$

$34(23.8 \%)$

$31(32.7 \%)$

86)

$38-48$

$43(30 \%)$

$36(32.7 \%) \quad 7(21.2 \%)$

$49-60$

$34(23.8 \%)$

$26(26.3 \%)$

$32(22.4 \%)$

17(15.5\%)

$8(24.2 \%)$

$>60$

$66(46.2 \%)$

$52(47.3 \%)$

$14(42.4 \%)$

$77(53.8 \%)$

$58(52.7 \%)$

$19(57.6 \%)$

\section{Smoking}

No

129(90.2\%)

100

$29(87.9 \%)$

$(90.9 \%)$

$13(9.1 \%) \quad 9(8.2 \%)$

$4(12.1 \%)$

Yes

$1(0.7 \%)$

$1(0.9 \%)$

$0(0 \%)$

$<0.05$

Unknown

Contact history

The history of contact with confirmed patients

$60(42.0 \%) \quad 46(41.8 \%) \quad 14(42.4 \%)$

The history of contact with epidemic area

$70(49.0 \%)$

$54(49.1 \%)$

$16(48.5 \%)$

Unknown

$9(6.3 \%)$

$6(5.5 \%)$

$3(9.1 \%)$

Both

$4(2.8 \%)$

$4(3.6 \%)$

$0(0 \%)$

\section{Temperature $\left({ }^{\circ} \mathrm{C}\right)$}

$<37.5$

$1(0.7 \%)$

$1(0.9 \%)$

$0(0 \%)$

$37.5-38$

$7(4.9 \%)$

$7(6.4 \%)$

$0(0 \%)$

$38-38.5$

$39(27.3 \%)$

$29(26.4 \%)$

$10(30.3 \%)$

$38.5-39$

$26(18.2 \%) \quad 19(17.3 \%) \quad 7(21.2 \%)$




\begin{tabular}{|c|c|c|c|c|}
\hline & \multirow{2}{*}{$\begin{array}{l}\text { Overall } \\
(n=143)\end{array}$} & \multicolumn{3}{|c|}{ Disease severity } \\
\hline & & $\begin{array}{l}\text { Non-severe } \\
(n=110)\end{array}$ & $\begin{array}{l}\text { Severe } \\
(n=33)\end{array}$ & $\begin{array}{l}\text { P- } \\
\text { Value }\end{array}$ \\
\hline $39-40$ & $32(22.4 \%)$ & $20(18.2 \%)$ & $12(36.4 \%)$ & - \\
\hline$>40$ & $1(0.7 \%)$ & $1(0.9 \%)$ & $0(0 \%)$ & - \\
\hline Unknown & 37 (25.9\%) & $33(30.0 \%)$ & $4(12.1 \%)$ & - \\
\hline \multicolumn{5}{|l|}{ Clinical symptoms } \\
\hline Fever & $105(73.4 \%)$ & $76(53.1 \%)$ & $29(20.1 \%)$ & \\
\hline Coughing of phlegm & $41(28.7 \%)$ & $30(27.3 \%)$ & $11(33.3 \%)$ & \\
\hline Dry cough & $42(29.4 \%)$ & $30(27.3 \%)$ & $12(36.4 \%)$ & \\
\hline Yellow sputum & $8(5.6 \%)$ & $6(5.5 \%)$ & $2(6.1 \%)$ & \\
\hline Sore throat & $15(10.5 \%)$ & $12(10.9 \%)$ & $3(9.1 \%)$ & \\
\hline Nasal obstruction & $8(5.6 \%)$ & $8(7.3 \%)$ & $0(0 \%)$ & \\
\hline Muscle soreness & $14(9.8 \%)$ & $11(10.0 \%)$ & $3(9.1 \%)$ & \\
\hline Weak & $37(25.9 \%)$ & $31(28.2 \%)$ & $6(18.2 \%)$ & \\
\hline Chest distress & $25(17.5 \%)$ & $17(15.5 \%)$ & $8(24.2 \%)$ & \\
\hline \multicolumn{5}{|l|}{ Coexisting disorders } \\
\hline hypertension & $20(14.0 \%)$ & $15(13.6 \%)$ & $5(15.2 \%)$ & \\
\hline diabetes & $12(8.4 \%)$ & $7(6.4 \%)$ & $5(15.2 \%)$ & \\
\hline COPD & $3(2.1 \%)$ & $2(1.8 \%)$ & $1(3.0 \%)$ & \\
\hline \multicolumn{5}{|c|}{ The incubation period(days) } \\
\hline$<3$ & $3(2.1 \%)$ & $3(2.7 \%)$ & $0(0 \%)$ & \\
\hline $3-6$ & $10(7 \%)$ & $9(7.3 \%)$ & $1(3.0 \%)$ & \\
\hline $6-9$ & $10(7 \%)$ & $9(8.1 \%)$ & $1(3.0 \%)$ & \\
\hline $9-12$ & $6(4.2 \%)$ & $3(2.7 \%)$ & $3(9.1 \%)$ & \\
\hline$>15$ & $4(2.8 \%)$ & $4(3.6 \%)$ & $0(0 \%)$ & \\
\hline Unknown & $110(76.9 \%)$ & 82 (74.5\%) & $28(84.8 \%)$ & \\
\hline \multicolumn{5}{|l|}{ Treatment delay } \\
\hline$<1$ & $49(34.3 \%)$ & 8 (24.2\%) & 41 (37.3\%) & \\
\hline
\end{tabular}




\begin{tabular}{|lllll|}
\hline & Overall & \multicolumn{2}{l|}{ Disease severity } & \\
\cline { 5 - 5 } & $(\mathbf{n = 1 4 3 )}$ & $\begin{array}{l}\text { Non-severe } \\
(\mathbf{n = 1 1 0 )}\end{array}$ & $\begin{array}{l}\text { Severe } \\
(\mathbf{n = 3 3 )}\end{array}$ & Palue \\
\hline $1-2$ & & $8(24.2 \%)$ & $32(29.1 \%)$ \\
\hline $2-5$ & $40(28.0 \%)$ & $16(14.5 \%)$ \\
\hline $5-10$ & $22(15.4 \%)$ & $6(18.2 \%)$ & $9(8.2 \%)$ \\
\hline$>10$ & $16(11.2 \%)$ & $7(21.2 \%)$ & $0(0 \%)$ \\
\hline Unknown & $2(1.4 \%)$ & $2(6.1 \%)$ & $12(10.9 \%)$ \\
\hline
\end{tabular}


Table 2

Laboratory Findings of Patients With COVID-19 on Admission to Hospital

\begin{tabular}{|c|c|c|c|c|c|}
\hline & $\begin{array}{l}\text { Normal } \\
\text { Range }\end{array}$ & $\begin{array}{l}\text { Overall } \\
(n=123)\end{array}$ & $\begin{array}{l}\text { Non- } \\
\text { Severe } \\
(n=95)\end{array}$ & Severe & P-value \\
\hline Lymphocyte count, $\times 10^{\wedge} 9 / \mathrm{L}$ & $1.1-3.2$ & $\begin{array}{l}1.2( \pm \\
0.52)\end{array}$ & $\begin{array}{l}1.3( \pm \\
0.52)\end{array}$ & $\begin{array}{l}0.91( \pm \\
0.43)\end{array}$ & $<0.001$ \\
\hline$<1.1 * 10^{\wedge} 9$ & & $\begin{array}{l}43 \\
(34.96 \%)\end{array}$ & $\begin{array}{l}27 \\
(28.42 \%)\end{array}$ & $\begin{array}{l}16 \\
(57.14 \%)\end{array}$ & \\
\hline $1.1 \sim 3.2 * 10^{\wedge} 9$ & & $\begin{array}{l}67 \\
(54.47 \%)\end{array}$ & $\begin{array}{l}60 \\
(63.16 \%)\end{array}$ & $7(25 \%)$ & \\
\hline unknown & & $\begin{array}{l}13 \\
(10.57 \%)\end{array}$ & $8(8.42 \%)$ & $\begin{array}{l}5 \\
(17.86 \%)\end{array}$ & $<1^{\star} 10^{\wedge} 9$ \\
\hline $\begin{array}{l}\text { Erythrocyte sedimentation rate } \\
\text { (ESR), umol/L }\end{array}$ & $59-104$ & $38( \pm 24)$ & $35( \pm 24)$ & $47( \pm 24)$ & 0.0615 \\
\hline$<59$ & & $68(55 \%)$ & $56(59 \%)$ & $12(43 \%)$ & 0.298 \\
\hline $59 \sim 104$ & & $12(10 \%)$ & $8(8 \%)$ & $4(14 \%)$ & \\
\hline Unknown & & $43(35 \%)$ & $31(33 \%)$ & $12(43 \%)$ & \\
\hline Blood glucose, mmol/L & $3.9-6.11$ & $13( \pm 53)$ & $14( \pm 60)$ & $9.1( \pm 5.1)$ & 0.476 \\
\hline $3.9 \sim 6.11$ & & $44(36 \%)$ & $36(38 \%)$ & $8(29 \%)$ & 0.599 \\
\hline$>6.11$ & & $62(50 \%)$ & $47(49 \%)$ & $15(54 \%)$ & \\
\hline Unknown & & $17(14 \%)$ & $12(13 \%)$ & $5(18 \%)$ & \\
\hline Aspartate aminotransferase, $\mathrm{U} / \mathrm{L}$ & $15-40$ & $30( \pm 17)$ & $26( \pm 11)$ & $40( \pm 28)$ & 0.0265 \\
\hline $15 \sim 40$ & & $91(74.0 \%)$ & $73(80.2 \%)$ & $18(19.8 \%)$ & \\
\hline$>40$ & & $17(13.8 \%)$ & $10(57.8 \%)$ & $7(42.2 \%)$ & \\
\hline Creatine kinase (CK), U/L & $38-285$ & $99( \pm 93)$ & $83( \pm 69)$ & $\begin{array}{l}150( \pm \\
130)\end{array}$ & 0.0262 \\
\hline $38 \sim 285$ & & $85(69 \%)$ & $68(72 \%)$ & $17(61 \%)$ & 0.54 \\
\hline$>285$ & & $13(11 \%)$ & $9(9 \%)$ & $4(14 \%)$ & \\
\hline Unknown & & $25(20 \%)$ & $18(19 \%)$ & $7(25 \%)$ & \\
\hline Lactic acid dehydrogenase, U/L & $80-285$ & $\begin{array}{l}240( \pm \\
140)\end{array}$ & $\begin{array}{l}200( \pm \\
100)\end{array}$ & $\begin{array}{l}350( \pm \\
180)\end{array}$ & $<0.001$ \\
\hline $80 \sim 285$ & & $72(59 \%)$ & $65(68 \%)$ & $7(25 \%)$ & $<0.001$ \\
\hline$>285$ & & $21(17 \%)$ & $8(8 \%)$ & $13(46 \%)$ & \\
\hline
\end{tabular}




\begin{tabular}{|c|c|c|c|c|c|}
\hline & $\begin{array}{l}\text { Normal } \\
\text { Range }\end{array}$ & $\begin{array}{l}\text { Overall } \\
(n=123)\end{array}$ & $\begin{array}{l}\text { Non- } \\
\text { Severe } \\
(n=95)\end{array}$ & Severe & P-value \\
\hline Unknown & & $30(24 \%)$ & $22(23 \%)$ & $8(29 \%)$ & \\
\hline Albumin, g/L & $40-55$ & $38( \pm 5.2)$ & $39( \pm 5.2)$ & $35( \pm 4.0)$ & 0.0175 \\
\hline$<40$ & & $16(13 \%)$ & $9(9 \%)$ & $7(25 \%)$ & 0.0116 \\
\hline $40 \sim 55$ & & $31(25 \%)$ & $29(31 \%)$ & $2(7 \%)$ & \\
\hline Unknown & & $76(62 \%)$ & $57(60 \%)$ & $19(68 \%)$ & \\
\hline $\mathrm{Na}, \mathrm{mmol} / \mathrm{L}$ & $137-147$ & $130( \pm 22)$ & $130( \pm 25)$ & $\begin{array}{l}140( \pm \\
3.7)\end{array}$ & 0.17 \\
\hline$<137$ & & $37(30 \%)$ & $29(31 \%)$ & $8(29 \%)$ & 0.78 \\
\hline $137 \sim 147$ & & 69 (56\%) & $54(57 \%)$ & $15(54 \%)$ & \\
\hline Unknown & & $17(14 \%)$ & $12(13 \%)$ & $5(18 \%)$ & \\
\hline $\mathrm{Mg}, \mathrm{mmol} / \mathrm{L}$ & $0.75-1.02$ & $3.8( \pm 17)$ & $4.7( \pm 19)$ & $\begin{array}{l}0.89( \pm \\
0.096)\end{array}$ & 0.0829 \\
\hline$<0.75$ & & $10(8 \%)$ & $8(8 \%)$ & $2(7 \%)$ & \\
\hline CRP, mg/L & $<8$ & $20( \pm 24)$ & $15( \pm 19)$ & $35( \pm 31)$ & 0.00592 \\
\hline $0 \sim 8$ & & $51(41 \%)$ & $44(46 \%)$ & $7(25 \%)$ & 0.132 \\
\hline$>8$ & & $51(41 \%)$ & $36(38 \%)$ & 15 (54\%) & \\
\hline Unknown & & $21(17 \%)$ & 15 (16\%) & $6(21 \%)$ & \\
\hline $\begin{array}{l}\text { Glomerular filtration rate (GFR), } \\
\mathrm{ml} / \mathrm{min}\end{array}$ & NA & $91( \pm 21)$ & $94( \pm 19)$ & $82( \pm 26)$ & 0.051 \\
\hline Amyloid A & $0-10$ & $\begin{array}{l}250( \pm \\
360)\end{array}$ & $\begin{array}{l}190( \pm \\
330)\end{array}$ & $\begin{array}{l}430( \pm \\
420)\end{array}$ & 0.0184 \\
\hline $0 \sim 10$ & & $21(17 \%)$ & $19(20 \%)$ & $2(7 \%)$ & \\
\hline$>10$ & & 79 (64\%) & $59(62 \%)$ & $20(74 \%)$ & \\
\hline fibrinogen, $s$ & $2.0-4.0$ & $3.9( \pm 1.4)$ & $3.7( \pm 1.4)$ & $4.5( \pm 1.3)$ & 0.0153 \\
\hline$>4$ & & $30(24 \%)$ & $17(18 \%)$ & $13(46 \%)$ & \\
\hline $0 \sim 4$ & & 60 (49\%) & $51(54 \%)$ & $9(32 \%)$ & \\
\hline D dimer level, g/L & $0-0.5$ & $\begin{array}{l}0.40( \pm \\
0.59)\end{array}$ & $\begin{array}{l}0.35( \pm \\
0.58)\end{array}$ & $\begin{array}{l}0.54( \pm \\
0.63)\end{array}$ & 0.25 \\
\hline $\mathrm{PaO} 2, \mathrm{mmHg}$ & $83-108$ & $94( \pm 31)$ & $99( \pm 33)$ & $83( \pm 24)$ & 0.0145 \\
\hline
\end{tabular}




\begin{tabular}{|c|c|c|c|c|c|}
\hline & $\begin{array}{l}\text { Normal } \\
\text { Range }\end{array}$ & $\begin{array}{l}\text { Overall } \\
(n=123)\end{array}$ & $\begin{array}{l}\text { Non- } \\
\text { Severe } \\
(n=95)\end{array}$ & Severe & P-value \\
\hline$<83$ & & $36(29 \%)$ & $23(24 \%)$ & $13(46 \%)$ & 0.143 \\
\hline $83 \sim 108$ & & $35(28 \%)$ & $28(29 \%)$ & $7(25 \%)$ & \\
\hline$>108$ & & $21(17 \%)$ & $18(19 \%)$ & $3(11 \%)$ & \\
\hline Unknown & & $31(25 \%)$ & $26(27 \%)$ & $5(18 \%)$ & \\
\hline Oxygen concentration, \% & NA & $26( \pm 13)$ & $25( \pm 14)$ & $27( \pm 11)$ & 0.585 \\
\hline $0 \sim 21$ & & $61(50 \%)$ & $51(54 \%)$ & $10(36 \%)$ & 0.0131 \\
\hline$>21$ & & $31(25 \%)$ & $18(19 \%)$ & $13(46 \%)$ & \\
\hline Unknown & & $31(25 \%)$ & $26(27 \%)$ & $5(18 \%)$ & \\
\hline Myoglobin, ng/ml, & $12-75$ & $46( \pm 56)$ & $35( \pm 41)$ & $84( \pm 80)$ & 0.0251 \\
\hline$<12$ & & $7(6 \%)$ & $6(6 \%)$ & $1(4 \%)$ & \\
\hline $12 \sim 75$ & & $54(44 \%)$ & $45(47 \%)$ & $9(32 \%)$ & \\
\hline$>75$ & & $12(10 \%)$ & $5(5 \%)$ & $7(25 \%)$ & \\
\hline IL-10, pg/ml & $0.1-5.0$ & $5.2( \pm 5.2)$ & $4.0( \pm 2.5)$ & $9.4( \pm 9.2)$ & 0.0247 \\
\hline $0 \sim 5$ & & $56(46 \%)$ & $49(52 \%)$ & $7(25 \%)$ & \\
\hline$>5$ & & $28(23 \%)$ & $17(18 \%)$ & $11(39 \%)$ & \\
\hline CD3 absolute value, /uL & $\begin{array}{l}770 \sim \\
2041\end{array}$ & $\begin{array}{l}720( \pm \\
450)\end{array}$ & $\begin{array}{l}790( \pm \\
460)\end{array}$ & $\begin{array}{l}490( \pm \\
330)\end{array}$ & 0.0245 \\
\hline$<770$ & & $28(23 \%)$ & $19(20 \%)$ & $9(32 \%)$ & \\
\hline $770 \sim 2041$ & & $17(14 \%)$ & $15(16 \%)$ & $2(7 \%)$ & \\
\hline CD4 absolute value, $/ \mu \mathrm{L}$ & $414-1123$ & $\begin{array}{l}440( \pm \\
260)\end{array}$ & $\begin{array}{l}490( \pm \\
260)\end{array}$ & $\begin{array}{l}290( \pm \\
200)\end{array}$ & 0.0149 \\
\hline$<414$ & & $26(21 \%)$ & $18(19 \%)$ & $8(29 \%)$ & \\
\hline $414-1123$ & & $19(15 \%)$ & $16(17 \%)$ & $3(11 \%)$ & \\
\hline CD8 absolute value $-/ \mu \mathrm{L}$ & $238-874$ & $\begin{array}{l}290( \pm \\
180)\end{array}$ & $\begin{array}{l}330( \pm \\
180)\end{array}$ & $\begin{array}{l}190( \pm \\
130)\end{array}$ & 0.00928 \\
\hline$<238$ & & $22(18 \%)$ & $15(16 \%)$ & $7(25 \%)$ & \\
\hline $238 \sim 874$ & & $23(19 \%)$ & $19(20 \%)$ & $4(14 \%)$ & \\
\hline
\end{tabular}


Table 3

CT Manifestations of Patients Infected With COVID-19 Admission to Hospital

\begin{tabular}{|c|c|c|c|c|}
\hline & \multirow{2}{*}{$\begin{array}{l}\text { Overall } \\
(n=138)\end{array}$} & \multirow{2}{*}{$\begin{array}{l}\text { Non- Severe } \\
(n=104)\end{array}$} & \multirow{2}{*}{$\begin{array}{l}\text { Severe } \\
(n=34)\end{array}$} & \multirow[t]{2}{*}{ P-value } \\
\hline & & & & \\
\hline \multicolumn{5}{|l|}{ Distribution characteristics } \\
\hline Peripheral or subpleural & $135(98 \%)$ & $102(98 \%)$ & $33(97 \%)$ & \\
\hline Middle or inner band & $37(27 \%)$ & $21(20 \%)$ & $16(47 \%)$ & 0.00441 \\
\hline both & $34(25 \%)$ & 19(18.3) & $15(44 \%)$ & \\
\hline \multicolumn{5}{|l|}{ Shape } \\
\hline Paving stone shape & $21(32 \%)$ & $15(15 \%)$ & $6(18 \%)$ & \\
\hline Mass & $51(81 \%)$ & $35(34 \%)$ & $16(47 \%)$ & \\
\hline Patchy & $24(40 \%)$ & $16(16 \%)$ & $8(24 \%)$ & \\
\hline Strip shape & $7(9 \%)$ & $6(6 \%)$ & $1(3 \%)$ & \\
\hline Honeycomb shape & $8(12 \%)$ & $6(6 \%)$ & $2(6 \%)$ & \\
\hline Small patchy & $58(68 \%)$ & $52(50 \%)$ & $6(18 \%)$ & \\
\hline Lung segment shape & $4(12 \%)$ & 0 & $4(12 \%)$ & \\
\hline Irregular & $4(12 \%)$ & 0 & $4(12 \%)$ & \\
\hline Nodular & $4(3.88 \%)$ & $4(3.88 \%)$ & 0 & \\
\hline \multicolumn{5}{|l|}{ Density } \\
\hline Ground glass density & $37(27 \%)$ & $30(29 \%)$ & $7(21 \%)$ & 0.325 \\
\hline Mixed Density & $87(63 \%)$ & $62(60 \%)$ & $25(74 \%)$ & \\
\hline Solid Density & $14(10 \%)$ & $12(12 \%)$ & $2(6 \%)$ & \\
\hline \multicolumn{5}{|l|}{ The edge } \\
\hline clear & $21(15 \%)$ & $16(15 \%)$ & $5(15 \%)$ & \\
\hline unclear & $117(85 \%)$ & $88(85 \%)$ & $29(85 \%)$ & \\
\hline \multicolumn{5}{|l|}{ CT signs } \\
\hline consolidation & $66(48 \%)$ & $43(41 \%)$ & $23(68 \%)$ & 0.0136 \\
\hline bronchial inflation & $63(46 \%)$ & $43(41 \%)$ & $20(59 \%)$ & 0.0015 \\
\hline Bronchiectasis & $65(47 \%)$ & $41(39 \%)$ & $24(71 \%)$ & 0.00305 \\
\hline Blood vessels penetrated the lesion & $57(41 \%)$ & $42(40 \%)$ & $15(44 \%)$ & 0.855 \\
\hline
\end{tabular}




\begin{tabular}{|c|c|c|c|c|}
\hline & \multirow{2}{*}{$\begin{array}{l}\text { Overall } \\
(n=138)\end{array}$} & \multirow{2}{*}{$\begin{array}{l}\text { Non-Severe } \\
(n=104)\end{array}$} & \multirow{2}{*}{$\begin{array}{l}\text { Severe } \\
(n=34)\end{array}$} & \multirow[t]{2}{*}{ P-value } \\
\hline & & & & \\
\hline distribution along the vascular bundle & $83(60 \%)$ & $64(62 \%)$ & $19(56 \%)$ & 0.702 \\
\hline Adjacent vascular widening & $72(52 \%)$ & $55(53 \%)$ & $17(50 \%)$ & 0.925 \\
\hline septal thickening & $58(42 \%)$ & $41(39 \%)$ & $17(50 \%)$ & 0.376 \\
\hline fibrosis & $42(30 \%)$ & $29(28 \%)$ & $13(38 \%)$ & 0.081 \\
\hline \multicolumn{5}{|l|}{ Accompanying signs } \\
\hline \multicolumn{5}{|l|}{ cavity or calcification } \\
\hline Non & 137(99\%) & $103(99 \%)$ & $34(100 \%)$ & \\
\hline Yes & $1(1 \%)$ & $1(1 \%)$ & $0(0 \%)$ & \\
\hline \multicolumn{5}{|l|}{ lymphadenectasis } \\
\hline Non & $134(97 \%)$ & $102(98 \%)$ & $32(94 \%)$ & 0.545 \\
\hline Yes & $4(3 \%)$ & $2(2 \%)$ & $2(6 \%)$ & \\
\hline \multicolumn{5}{|l|}{ pleural effusion } \\
\hline Non & $135(98 \%)$ & $103(99 \%)$ & $32(94 \%)$ & 0.303 \\
\hline Yes & $3(2 \%)$ & $1(1 \%)$ & $2(6 \%)$ & \\
\hline \multicolumn{5}{|l|}{ chronic bronchitis } \\
\hline Non & $132(96 \%)$ & $100(96 \%)$ & $32(94 \%)$ & 0.983 \\
\hline Yes & $6(4 \%)$ & $4(4 \%)$ & $2(6 \%)$ & \\
\hline \multicolumn{5}{|l|}{ emphysema or pulmonary bullous } \\
\hline Non & $133(96 \%)$ & $101(97 \%)$ & $32(94 \%)$ & 0.777 \\
\hline Yes & $5(4 \%)$ & $3(3 \%)$ & $2(6 \%)$ & \\
\hline
\end{tabular}


Table 4

Treatments and Clinical Outcomes

\begin{tabular}{|lllll|}
\hline & Overall & Non Severe & Severe & \multirow{2}{*}{ P-value } \\
\cline { 2 - 4 } & $(\mathbf{n = 1 4 3 )}$ & $(\mathbf{n = 1 1 0 )}$ & $(\mathbf{n = 3 3 )}$ & \\
\hline Treatment & & & & \\
\hline Oxygen-inhalation & $32(56 \%)$ & $59(53 \%)$ & $22(67 \%)$ & 0.0463 \\
\hline Antiviral & $87(61 \%)$ & $71(65 \%)$ & $16(49 \%)$ & 0.181 \\
\hline Intravenous Glucocorticoids & $16(11 \%)$ & $5(5 \%)$ & $11(33 \%)$ & $<0.001$ \\
\hline Gamma globulin & $9(6 \%)$ & $3(3 \%)$ & $6(18 \%)$ & $<0.001$ \\
\hline Antibiotics & $21(15 \%)$ & $15(14 \%)$ & $6(18 \%)$ & 0.843 \\
\hline Noninvasive ventilation & $29(20 \%)$ & $25(23 \%)$ & $4(12 \%)$ & 0.209 \\
\hline Invasive mechanical ventilation & $1(1 \%)$ & $0(0 \%)$ & $1(3 \%)$ & \\
\hline ECMO & 0 & 0 & 0 & \\
\hline Clinic Outcome & & & & \\
\hline Discharge from hospital & $143(100 \%)$ & $110(100 \%)$ & $33(100 \%)$ & \\
\hline Die & $0(0 \%)$ & $0(0 \%)$ & $0(0 \%)$ & \\
\hline
\end{tabular}

\section{Laboratory confirmation}

All cases were confirmed by Zhejiang Center for Disease Control (CDC). The nucleic acid extraction was carried out with the kit (Biogas) recommended by the Chinese center for disease control and Control (CDC). RT-PCR assays were performed in accordance with the protocol established by the WHO, and Nucleic acid sequencing was performed using NGS (high throughput sequencing) technology.

\section{Statistical analysis}

We applied the statistical software of EXCEL (version 2019) and R software (version 3.6.1) for data processing and analysis. Continuous variables are expressed as mean (standard deviation) or median (quartile spacing or range), and the classification variables were expressed by count (percentage). Chisquare test was used for comparison between groups. $\mathrm{P}<0.05$ was considered statistically significant.

\section{Results}

Demographic characteristics. 
As of March 11, 2020, a total of 146 patients were confirmed in the four hospitals and transferred to the isolation hospital for treatment. We obtained basic information, clinical data and CT images of 143 patients (96.6\%), among whom 66 were females (46.2\%) and 77 males (53.8\%). Demographic and clinical characteristics of the patients are shown in Table 1. On admission, the degree of severity of COVID-19 was categorized as non-severe in 110 patients and severe in 33 patients. Of all patients, 60 patients $(42.0 \%)$ had contact with a confirmed patient, 70 patients $(49.0 \%)$ had contact with a person in the Wuhan area, 4 patients $(2.8 \%)$ had contact with both, and 9 patients $(6.3 \%)$ had an unclear contact history. The median age of patients was 47 years old (range $4-86$ ), 56.8\% were aged $30-60$ years old.

The median age of the non-severe patients was 44.5 years old (range 4-80), while the median age of the severe patients was 55.0 years old (range $27-86)$, and the difference was statistically significant $(\mathrm{P}<$ $0.05)$. The number of young patients (<38 years old) in the non-severe group $(32.7 \%)$ was higher than that in the severe group $(0.9 \%)$, and the difference was statistically significant $(P<0.05)$. The proportion of old patients (> 60 years old) in the severe group (45.5\%) was greater than that in the non-severe group (15.5\%), and the difference was statistically significant $(P<0.05) .129$ patients $(90.2 \%)$ had a history of smoking, including 100 patients (90.9\%) in the non-severe group and 29 patients (87.9\%) in the severe group, with no statistically significant difference.

The mean incubation period was 6.9 days (SD3.472) and the mean treatment delay period was 3.0 days (SD2.631). Fever (73.4\%) was the most common symptom. And a total of 91 patients $(63.6 \%)$ developed cough, with sputum (28.7\%), yellow sputum (5.6\%) and dry cough $(29.4 \%)$. Sore throat $(10.5 \%)$, Nasal congestion (5.6\%), muscle soreness (9.8\%), and chest tightness (17.5\%) were relatively few. Among the overall population, $24.5 \%$ had at least one coexisting illness (e.g., hypertension and chronic obstructive pulmonary disease). The average body temperature of patients was $38.0^{\circ} \mathrm{C}(\mathrm{SD} 3.741)$, mainly in $37^{\circ} \mathrm{C}-39^{\circ} \mathrm{C}$, and only 1 case $(0.7 \%)$ had high fever $\left(>40^{\circ} \mathrm{C}\right)$.

\section{Differences in laboratory results between the two groups of patients}

Some of the patients were first admitted to the county hospital, and some laboratory tests were not carried out due to conditions. A total of 123 cases were included for laboratory examination results analysis, and those without such examination were recorded as UNKOWN. There were 28 cases in the severe group and 95 cases in the non-severe group. Table 2 shows the details of laboratory results of all cases. In the non-severe group, except for the increase of blood glucose $(14 \pm 60 \mathrm{mmol} / \mathrm{L})$, the decrease of serum albumin $(39 \pm 5.2 \mathrm{~g} / \mathrm{L})$, the decrease of erythrocyte sedimentation rate $(35 \pm 24 \mathrm{umol} / \mathrm{L})$, the decrease of serum sodium $(130 \pm 25) \mathrm{mmol} / \mathrm{L})$, the increase of CRP $(15 \pm 19 \mathrm{mg} / \mathrm{L})$, and the increase of amyloid $A(190 \pm 330)$. All other test results were within the normal range.

In the severe group, the lymphocyte count decreased $\left((0.91 \pm 0.43)^{\star} 10^{\wedge} 9\right)$, the erythrocyte sedimentation rate $(38 \pm 24 \mathrm{umol} / \mathrm{L})$ decreased, the blood glucose $(13 \pm 53 \mathrm{mmol} / \mathrm{L})$ increased, the lactate dehydrogenase $(350 \pm 180 \mathrm{U} / \mathrm{L})$ increased, the serum albumin $(130 \pm 25 \mathrm{~g} / \mathrm{L})$ decreased, the CPR $(35 \pm$ $31 \mathrm{mg} / \mathrm{L})$ increased, and the glomerular filtration rate $(82 \pm 26 \mathrm{ml} / \mathrm{min})$ decreased. Amyloid protein $A$ $(430 \pm 420)$ increased, the fibrinogen detection value $(4.5 \pm 1.3 \mathrm{~s})$ increased, the $D$ dimer $(0.54 \pm 0.63 \mathrm{~g} / \mathrm{L})$ 
increased, the $\mathrm{PH}(7.8 \pm 2.0)$ increased, the arterial blood oxygen partial pressure $(83( \pm 24) \mathrm{mm} / \mathrm{Hg})$ decreased. And the calcitonin original $(0.086 \pm 0.090 \mathrm{ug} / \mathrm{L})$ level is reduced, the myoglobin $(84 \pm$ $80 \mathrm{ng} / \mathrm{ml}), \mathrm{IL}-10(9.4 \pm 9.2 \mathrm{pg} / \mathrm{ml}), \mathrm{C} 1 \mathrm{q}(240 \pm 38 \mathrm{mg} / \mathrm{L})$ increased, the PT $(16 \pm 19 \mathrm{~s})$ increased, the absolute value of CD3 $(490 \pm 330 / \mathrm{uL})$ increased, the totally value of CD3 $(56 \pm 13 \%)$ decreased, the absolute value of CD4 $(290 \pm 200 / \mathrm{uL})$ decreased, and the absolute value of CD8 $(190 \pm 130 / \mathrm{uL})$ decreased. Other test results were within the normal range.

In addition, the result of following laboratory test items were different, such as aspartate transaminase, creatine kinase and lactate dehydrogenase, serum albumin, CPR, glomerular filtration rate, amyloid protein A, fibrinogen, calcitonin level, oxygen partial pressure, red protein, IL -10 , the absolute value of $\mathrm{CD} 3$, and the absolute value of CD4, the absolute value of CD8. The difference between the two groups was statistically significant $(P<0.05)$ (table2).

\section{CT manifestations of the two groups}

Five (3\%) CT images were unable to be evaluated due to poor respiratory artifact quality. Images of 138 (97\%) patients at admission were obtained. Among them, 34 patients were severe (31\%) and 104 patients were non-severe (75\%). The lesions of 135 patients $(98 \%)$ mainly distributed in the external or subpleural of lung, 37 patients (27\%) in the middle or inner zone of lung, and 34 patients (25\%) in both. Among them, the lesions in the middle or inner band were more common seen in non-severe patients (47\% vs $20 \%$ ), and the difference was statistically significant $(P=0.004)$. Mass $(81 \%)$ was the most common lesion shape, followed by patchy $(68 \%)$. The shapes of lung segments and irregular were seen only in the severe patients, while the nodular shape was seen only in the non-severe patients. Most of the lesions presented with mixed density $(63 \%)$.

In $60 \%$ of the CT images, the lesion was distributed along the pulmonary bronchial tree, and in $62 \%$ of the CT images, the lesion was adjacent to vasodilation. There were $52 \%$ CT images showing interlobular septal thickening, and fibrous foci were present in $30 \%$ of the CT images. Other concomitant signs such as cavitation or calcification (1\%), enlarged lymph nodes (3\%), pleural effusion ( $2 \%)$, chronic bronchitis (4\%), emphysema or pulmonary bullous (4\%) were rare. Consolidation ( $68 \%$ vs $41 \%$ ), bronchial inflation signs $(59 \%$ vs $41 \%)$, and bronchiectasis ( $71 \%$ vs $39 \%$ ) were more common in the severe group, and the difference was statistically significant $(P<0.05)$.

All the discharged patients (61/143) showed obvious absorption of lesions on CT. Figure 1 shows the changes on lesions on CT of a discharged patient from admission to discharge.

\section{Treatment and clinical outcomes.}

81 patients (56\%) received oxygen therapy and 95 (65\%) received antiviral therapy. Antiviral therapy drugs were interferon, Lopinavir and ritonavir, Abidor, Lianhua qingwen capsules, Oseltamivir and so on. 16 patients $(11 \%)$ were treated with intravenous glucocorticoids, 11 of whom were in the severe group. 9 patients $(6 \%)$ were treated with gamma globulin and $21(15 \%)$ with antibiotics. Noninvasive respiratory 
support was used in 29 patients (20\%) and invasive respiratory support was used in 1 severe patient. As of March 11, 2020, the final date of follow-up, all patients were discharged from the hospital, with no deaths.

\section{Discussions}

In the early stage of novel coronavirus outbreak or coronavirus disease outbreak without good research and understanding, the diagnosis and severity of COVID-19 were mainly based on clinical symptoms and imaging manifestations. In order to provide the clinical characteristics and imaging findings of COVID-19 infections in cities outside Wuhan, this study collected 143 patients confirmed by laboratory test from four hospitals in Taizhou, including 33 severe patients and non-severe 110 patients.

$42 \%$ of the patients have been living in Wuhan for a long time or have been to Wuhan or had contact with people returning from Wuhan. This result was similar to that of other studies[23]. Our study also found that the average age of COVID-19 infected patients was 47.0(range 4-86) years old, which was similar to findings of previous study[23]. The results of this study showed no statistical difference between men and women, but the study of Chen Nanshan et al[20] found that the proportion of men was higher than that of women, but the number of their study was only 99 , so more data are needed to confirm the accuracy of this result. Most of the patients (62.3\%) went to the hospital within 2 days of symptom onset. The average incubation period in study by Weijie Guan et al. was 4 days (2-7 days) [23], while that was 5.2 days (4.1-7.0 days) by Li Qun et al. [24].Fever and cough were the most common clinical symptoms in all cases, with fever occurring in $73.4 \%$ and cough in $63.6 \%$. Compared with other study, our results were different. In the study of Guan Weijie et al.[23] 43.8\% patients were found to have fever at the first visit, but the number increased to $88.7 \%$ after hospitalization. In our study, of all patients with fever, more than half had mild to moderate fever $\left(37.5-39^{\circ} \mathrm{C}\right)$, and only a few had high fever $(23.1 \%)$.

The laboratory test results of both groups were abnormal to different degrees, especially in patients with severe diseases. Blood glucose, serum sodium, serum albumin, amyloid A, and CRP values were found to be outside the normal range in both groups. We suggest that the difference in laboratory results between the two groups, which can be used to assess the degree of illness to some extent. The accuracy still needs to be confirmed with larger data however. In addition, the possible explanation was that the COVID -19 , a novel virus, greatly triggers the body's innate immune response, adaptive response and specific immune response after entering the body through the respiratory tract[21]. Specific immune response depends primarily on $\mathrm{T}$ cells, and the critical protective role of $\mathrm{T}$ cell immune response in coronavirus infection has been well documented in several animal models[25].

In the early stage of the disease or in non-severe patients, the body's innate immune response and specific response can restrain the spread and clearance of the virus, just like other viruses invading the body, only increased blood glucose, accelerated CRP, increased amyloid A and other adaptive responses, as well as diluent serum albumin and blood sodium reduction. With the progress of the disease, despite the efforts of T cells to fight against the virus, CD4, CD8 and other lymphocytes were reduced in severe 
patients or in the later stage of the disease due to the virulence of the virus or the decline of the body's immunity. Possible, the mechanism was similar to that of CD4 cell reduction caused by HIV[26, 27]. These results reflect the body reach the maximum to clear the virus, so that this stage may result in decreased lung function, liver function, kidney function, and even blood clotting and heart function. The specific manifestations were reduced $\mathrm{PaO} 2$, increased transaminase, decreased glomerular filtration rate, increased PT and D dimer values, and increased myoglobin (Table 2). At the same time, if the treatments working well or the patient's resistance system recovery, the multiple organ dysfunction reversed. But if the disease continues to progress or measures are not effective, all organs will fail, especially the lung, even to death. In Dawei Wang's study of 138 inpatients in Wuhan, the mortality rate was 4.3\%[21], while $1.4 \%$ in another study[23].Accurate death rates require further statistics.

In all the cases in this study, only one 18-year-old patient presented no obvious imaging manifestations at the time of admission, and all the others showed imaging changes. Among the remaining 138 cases assessed with imaging changes, the lesions were more localized in Lung periphery (98\%), and only a few severe patients showed inner or middle band lesions. This result was similar to that of the Wenzhou case imaging study[28].The possible explanation was that the blood supply of the subpleural is less than that of intrapulmonary band and mediastinum band, with lymphatic reflux reduced, resulting in a relatively low virus clearance capacity. There were more patchy heterogeneous density shadows in patients. The possible explanation was that different exudate protein content would lead to different density on CT, resulting in a variety of shapes and density changes. In $60 \%$ of all patients, the lesions were distributed along the lung texture, suggesting that spread along the bronchi may be one of the mechanisms of the virus spread. In addition, CT signs such as consolidation, air bronchodilatation and bronchodilation were more common in the severe group, so we believe that these signs can be used to assess the severity of the disease.

Some limitations in our study. First, the earliest contact date between some patients and the potential source of infection was not clear, resulting in the accuracy of incubation period calculation was affected. Second, since the laboratory verification of the case was conducted by the Zhejiang CDC, Ct values of the patient's PCR examination at admission were not obtained. More complete data should include the patient's viral load or at least the Ct value. Third, this study was not a randomized study, and the difference in the number of cases between the two groups may affect the statistics.

In short, most of the patients in Taizhou were non-severe, and the majority had contact with a potential source of infection. Fever and cough were the most common initial symptoms. The laboratory examination of non-severe cases mainly focused on the abnormalities of inflammatory indicators such as accelerated CPR and elevated amyloid A, while patients in the severe group could also have dysfunction of other systems besides the respiratory system. Differences in the results of some laboratory items can be used to assess the severity of the disease, such as absolute values of CD3, CD4, CD8, and so on. Most of the CT imaging showed multifocal subpleural heterogeneous density patches. The disease was aggravated when there were signs of consolidation, bronchiectasis and air bronchodilatation on CT. 


\section{Conclusions}

Most of the cases of COVID-19 in Taizhou have mild symptoms and no death. In addition to clinical symptoms, some laboratory tests (such as absolute values of CD4 and CD8) and CT findings can be used to assess the severity of the disease.

\section{Abbreviations}

CT: Computed tomography; WHO: World Health Organization; SARS-CoV-2: Severe acute respiratory syndrome coronavirus 2; SARS-CoV: Severe acute respiratory syndrome coronavirus; MERS-CoV: Middle east respiratory syndrome coronavirus; PHEIC: Public Health Emergency of International Concern; COVID19: Coronavirus disease 2019; CDC: Center for Disease Control and Prevention; RT-PCR: reverse transcription polymerase chain reaction; NGS: high throughput sequencing PaCO2: Arterial blood oxygen partial pressure; RR: Respiratory Rate;FiO2:oxygen concentration; PH: potential of hydrogen;C1q: Human Complement Component C1q;IL-10: Interleukin-10;TCD3:Total cluster of differentiation 3;PT: Prothrombin time; CK: Creatinine kinase; LDH: Lactate dehydrogenase; CRP: C-reactive protein; HIV: human immunodeficiency virus; $C D 3 / 4 / 8$ : cluster of differentiation 3/4/8;

\section{Declarations}

\section{Ethical Approval}

This retrospective multi-center cohort study was approved by the ethics review committee of Taizhou Hospital, Sanmen People's Hospital, Wenling First People's Hospital and Tiantai People's Hospital. Given the urgency of the data, written informed consent was waived.

\section{Acknowledgments}

We thank Jingjing Li for her contribution to date collection for this study.

\section{Funding source}

None

\section{Conflicts of interest}

The authors decl that they have no competing interests.

\section{Consent to participate}


Given the urgency of the data, written informed consent was waived.

\section{Consent for publication}

All authors agreed to publish the manuscript in the Journal of Virology Journal

\section{Availability of data and material}

All datasets are presented in the main paper.

\section{Code availability}

Not applicable

\section{Authors' Contributions}

$\mathrm{YK}, \mathrm{SH}$ and WJ designed the study and took the lead in drafting the manuscript and interpreting, SL developed the statistical methods, Susu He, RZ, SY, RZ, RL, HL, ZZ were participated in the collection of experimental data. PP was assisted in the writing and correction of this manuscript. All authors read and approved the final manuscript for publication

\section{References}

1. Wang W, Tang J, Wei F. Updated understanding of the outbreak of 2019 novel coronavirus (2019nCoV) in Wuhan, China. J Med Virol [Internet]. 2020;0-3. Available from:

http://www.ncbi.nlm.nih.gov/pubmed/31994742

2. Hui DS, I Azhar E, Madani TA, Ntoumi F, Kock R, Dar O, et al. The continuing 2019-nCoV epidemic threat of novel coronaviruses to global health - The latest 2019 novel coronavirus outbreak in Wuhan, China. Int J Infect Dis. 2020;91:264-6.

3. Bassetti M, Vena A, Roberto Giacobbe D. The Novel Chinese Coronavirus (2019-nCoV) Infections: challenges for fighting the storm. Eur J Clin Invest. 2020;1-4.

4. Lu R, Zhao X, Li J, Niu P, Yang B, Wu H, et al. Genomic characterisation and epidemiology of 2019 novel coronavirus: implications for virus origins and receptor binding. Lancet [Internet]. Elsevier Ltd; 2020;395:565-74. Available from: http://dx.doi.org/10.1016/S0140-6736(20)30251-8

5. CHINA X. XINHUA. The world health organization named the pneumonia infected by the new coronavir. 2020; Available from: http://www.xinhuanet.com//2020-02/11/c_1125561343.htm. 2020;2020. 
6. WHO. Coronavirus. Accessed on 20 Jan 2020. Jan 11, 2020 [Internet]. 2020; Available from: https://www.who.int/healthtopics/coronavirus

7. Rothe C, Schunk M, Sothmann P, Bretzel G, Froeschl G, Wallrauch C, et al. Transmission of 2019nCoV Infection from an Asymptomatic Contact in Germany. N Engl J Med. 2020;2019-20.

8. Phan LT, Nguyen T V, Luong QC, Nguyen T V, Nguyen HT, Le HQ, et al. Importation and Human-toHuman Transmission of a Novel Coronavirus in Vietnam. N Engl J Med. 2020;

9. Chan JFW, Yuan S, Kok KH, To KKW, Chu H, Yang J, et al. A familial cluster of pneumonia associated with the 2019 novel coronavirus indicating person-to-person transmission: a study of a family cluster. Lancet [Internet]. Elsevier Ltd; 2020;395:514-23. Available from: http://dx.doi.org/10.1016/S0140-6736(20)30154-9

10. Wu JT, Leung K, Leung GM. Nowcasting and forecasting the potential domestic and international spread of the 2019-nCoV outbreak originating in Wuhan, China: a modelling study. Lancet [Internet]. Elsevier Ltd; 2020;6736. Available from: http://dx.doi.org/10.1016/S0140-6736(20)30260-9

11. CHINA D. DINGXIANG garden, doctor DINGXIANG, real-time status of new coronavirus pneumonia. :5. Available from: https://ncov.dxy.cn/ncovh5/view/pneumonia? from $=$ singlemessage\&isappinstalled $=0$

12. Ren W, Qu X, Li W, Han Z, Yu M, Zhou P, et al. Difference in Receptor Usage between Severe Acute Respiratory Syndrome (SARS) Coronavirus and SARS-Like Coronavirus of Bat Origin. J Virol [Internet]. 2008;82:1899 LP - 1907. Available from: http://jvi.asm.org/content/82/4/1899.abstract

13. Maier HJ, Bickerton E, Britton P. Coronaviruses: An Overview of Their Replication and Pathogenesis. Coronaviruses Methods Protoc. 2015;1-282.

14. Hemida MG, Elmoslemany A, Al-hizab F, Alnaeem A, Almathen F, Faye B, et al. Dromedary camels and the transmission of Middle East Respiratory Syndrome Coronavirus (MERS-CoV). Transbound Emerg Dis. 2018;64:344-53.

15. Kucharski AJ, Althaus CL. The role of superspreading in Middle East respiratory syndrome coronavirus (MERS-CoV) transmission. 2015;14-8.

16. Mackay IM, Arden KE. MERS coronavirus: Diagnostics, epidemiology and transmission. Virol J [Internet]. Virology Journal; 2015;12:1-21. Available from: http://dx.doi.org/10.1186/s12985-0150439-5

17. Azhar El, Hui DSC, Memish ZA, Drosten C, Zumla A. The Middle East Respiratory Syndrome (MERS). Infect Dis Clin North Am. 2019;33:891-905.

18. Lim J, Jeon S, Shin HY, Kim MJ, Seong YM, Lee WJ, et al. Case of the Index Patient Who Caused Tertiary Transmission of COVID-19 Infection in Korea: the Application of Lopinavir/Ritonavir for the Treatment of COVID-19 Infected Pneumonia Monitored by Quantitative RT-PCR. J Korean Med Sci [Internet]. The Korean Academy of Medical Sciences; 2020;35:e79-e79. Available from: https://pubmed.ncbi.nlm.nih.gov/32056407

19. Team C-19 NIRS. COVID-19, Australia: Epidemiology Report 2 (Reporting week ending 19:00 AEDT 8 February 2020). Commun Dis Intell [Internet]. Australia; 2020;44:10.33321/cdi.2020.44.14. Available 
from: https://pubmed.ncbi.nlm.nih.gov/32050080

20. Chen N, Zhou M, Dong X, Qu J, Gong F, Han Y, et al. Epidemiological and clinical characteristics of 99 cases of 2019 novel coronavirus pneumonia in Wuhan, China: a descriptive study. Lancet (London, England) [Internet]. Elsevier Ltd; 2020;6736:1-7. Available from: http://www.ncbi.nlm.nih.gov/pubmed/32007143

21. Wang D, Hu B, Hu C, Zhu F, Liu X, Zhang J, et al. Clinical Characteristics of 138 Hospitalized Patients with 2019 Novel Coronavirus-Infected Pneumonia in Wuhan, China. JAMA - J Am Med Assoc. 2020;1-9.

22. CHINA G office. General office of China health commission. New coronavirus diagnosis and treatment protocol (trial version 5). 1-3[2020-02-13]. Chinese J Tradit Chinese Med [Internet]. 2020;2011:1-9. Available from: http://kns.cnki.net/kcms/detail/11.2787.R.20200208.1034.002.html.

23. Guan W-J, Ni Z-Y, Hu Y, Liang W-H, Ou C-Q, He J-X, et al. Clinical Characteristics of Coronavirus Disease 2019 in China. N Engl J Med. 2020;1-13.

24. Li Q, Guan X, Wu P, Wang X, Zhou L, Tong Y, et al. Early Transmission Dynamics in Wuhan, China, of Novel Coronavirus-Infected Pneumonia. N Engl J Med. 2020;1-9.

25. Liu WJ, Zhao M, Liu K, Xu K, Wong G, Tan W, et al. T-cell immunity of SARS-CoV: Implications for vaccine development against MERS-CoV. Antiviral Res [Internet]. Elsevier B.V; 2017;137:82-92. Available from: http://dx.doi.org/10.1016/j.antiviral.2016.11.006

26. Akiyama H, Miller CM, Ettinger CR, Belkina AC, Snyder-Cappione JE, Gummuluru S. HIV-1 introncontaining RNA expression induces innate immune activation and T cell dysfunction. Nat Commun [Internet]. Springer US; 2018;9. Available from: http://dx.doi.org/10.1038/s41467-018-05899-7

27. Agosto LM, Henderson AJ. CD4+ T Cell Subsets and Pathways to HIV Latency. AIDS Res Hum Retroviruses. 2018;34:780-9.

28. Yang W, Cao Q, Qin L, Wang X, Cheng Z, Pan A, et al. Clinical characteristics and imaging manifestations of the 2019 novel coronavirus disease (COVID-19):A multi-center study in Wenzhou city, Zhejiang, China. J Infect [Internet]. Elsevier Ltd; 2020; Available from: http://www.sciencedirect.com/science/article/pii/S0163445320300992 NS -

\section{Figures}




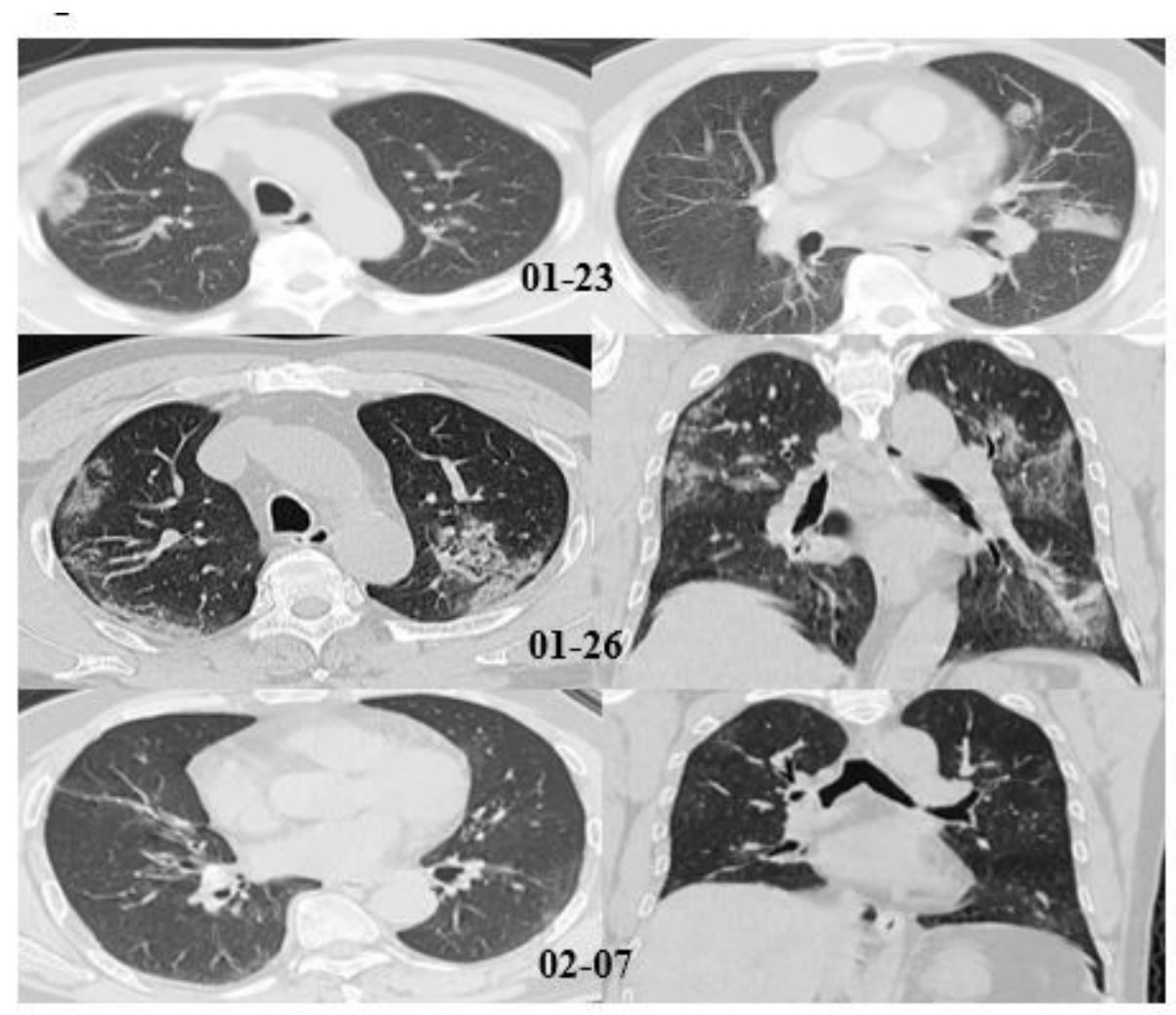

\section{Figure 1}

Chest CT of a male patient, 54 years old, living in Wuhan for a long time, he developed fever, herpes around the mouth with no cough on January 21 . He came to Taizhou hospital on January 23, and the result of RT-PCR test of throat swab was positive. He underwent a chest CT examination on January 23th $囚$ which showed multiple patchy, partial and mixed density shadows in both lungs. The lesion boundary in the tongue segment of the upper lobe of the left lung was blurred. Dilated segment bronchus was seen beside the lesion. The second time (January 26,2020) CT examination showed that the number of lesions increased significantly compared with the previous one, involving 5 lobes of the whole lung. The density of the lesions was uneven, varied in shape, and the boundary was blurred. The lesions were distributed along the lung texture and subpleural. One day before discharge (February 7,2020 ), the CT examination showed obvious absorption of the lesion, with a few small strips of patchy slightly highdensity shadows but still a few blurry patches. During the hospitalization, the patient had Aluvia as antiviral treatment and nasal spray of interferon, no oxygen therapy was performed since the oxygen saturation of the patient remained normal. 